therapeutic effect but better side-effect profiles than more traditional antipsychotics (Lader, 1999).

A 30-year-old patient with paranoid psychosis for 5 years and seizures for 12 years, described on average two generalised seizures a year, improving with valproate. His psychosis had been controlled with zuclopenthixol for 2 years. He had normal electroencephalograms (EEGs) in 1986 and 1998, including a sleep study while taking zuclopenthixol but not valproate. His psychosis relapsed secondary to noncompliance with medication and so zuclopenthixol $400 \mathrm{mg}$ twice weekly was recommenced. He improved, but owing to concerns over potential side-effects was changed to olanzapine $10 \mathrm{mg}$ daily. Over the next 3 months he suffered increasing seizures culminating in a generalised or tonic-clonic seizure resulting in bilateral humeral head fractures, one of which required internal fixation.

There was no metabolic or electrolyte disturbance. An EEG showed multifocal and generalised epileptiform discharges similar to those seen with clozapine, which are unusual for zuclopenthixol. They resolved on withdrawal of olanzapine and reinstitution of zuclopenthixol.

Conventional neuroleptics lower seizure threshold, yet this patient with a history of epilepsy had normal EEGs while on zuclopenthixol. Manufacturer's trials gave a seizure rate, similar to other antipsychotics, of $0.88 \%$ patients (product data sheet, Eli Lilly). However, other epileptogenic factors were present in these patients and also in two subsequent case reports involving olanzapine and seizures (Lee $e t a l$, 1999; Wyderski et al, 1999).

Our patient thus represents the strongest case to date implicating olanzapine alone in lowering seizure threshold, with objective EEG support.

Post-marketing surveillance and case reports are a useful early warning system for reporting side-effects, for example, sertindole with cardiotoxicity and more recently olanzapine with impaired glucose tolerance. This serves to remind all practitioners of the importance of considering a possibly underemphasised side-effect within the context of a newly introduced therapy. Olanzapine should be used cautiously in patients who have a history of seizures.

Lader, M. (1999) Some adverse effects of antipsychotics: prevention and treatment. Journal of Clinical Psychiatry, 60 (suppl. 12), 18-21.
Lee, J. W., Crismon, M. L. \& Dorson, P. G. (1999)

Seizure associated with olanzapine. Annals of Pharmacotherapy, 33, 554-556.

Wyderski, R. J., Starrett, W. G. \& Abou-Saif, A. (1999) Fatal status epilepticus associated with olanzapine therapy. Annals of Pharmacotherapy, 33 787-789.

J.Woolley, S. Smith Maudsley Hospital, Denmark Hill, London SE5 8AZ

\section{Olanzapine: concordant response in monozygotic twins with schizophrenia}

There is growing evidence that genetic variation in several neurotransmitter systems (e.g. serotonergic) may influence the clinical response to different psychopharmacological drugs (Arranz et al, 1998, 2000). A previous paper (Vojvoda et al, 1996) described the concordant clinical response of a pair of monozygotic twins with schizophrenia when treated with clozapine. Now we report on two monozygotic twins concordant for DSM-IV (American Psychiatric Association, 1994) schizophrenia whose clinical response to olanzapine was also concordant.

The twins are now 60 years old. Twin 1 developed her first psychotic symptoms at age 21 . Since then, she has been repeatedly admitted to hospital because of worsening of her psychotic symptoms, never returning to her premorbid level of functioning. She was treated with a wide variety of conventional antipsychotics, always with a poor response. Prior to her first psychotic breakdown, she suffered a seizure, and was treated with phenobarbital and valproate. At age 58 years she was started on olanzapine building up to a high dose ( $20 \mathrm{mg}$ daily) to control her symptoms. With this drug she had a good response (both in positive and negative psychotic symptoms) and an improvement in her level of functioning.

Twin 2 had her first psychotic episode and hospital admission at age 24. Subsequently, she was treated with different conventional antipsychotics as well as with clozapine, but never achieved a successful recovery. She needed several hospital treatments and suffered two seizures, with normal electroencephalogram while taking clozapine and levomepromazine, and agranulocytosis under clozapine treatment. Encouraged by her sister's response to olanzapine, she was treated with $20 \mathrm{mg}$ olanzapine daily. She showed a good response, soon improving in both positive and negative symptoms, and in her level of functioning. Each twin is now symptomfree, working and living unaided. Their response to olanzapine treatment has been similar both in intensity and in the pattern of symptoms that have improved. To our knowledge, this is the first report describing monozygotic twins with similar illness characteristics who showed a similar response to olanzapine treatment. Our finding supports the view that, as with clozapine, genetic factors may be important in predicting response to olanzapine and other antipsychotic drugs.

American Psychiatric Association (1994) Diagnostic and Statistical Manual of Mental Disorders (4th edn) (DSM-IV).Washington, DC: APA

Arranz, M. J., Munro, J., Sham, P., et al (1998) Metaanalysis of studies on genetic variation in $5-\mathrm{HT} 2 \mathrm{~A}$ receptors and clozapine response. Schizophrenia Research, 32, 93-99.

Birkett, J., et al (2000) Pharmacogenetic prediction of clozapine response. Lancet, 355, 1615-1616.

Vojvoda, D., Grimmell, K., Sernyak, M., et al (1996)

Monozygotic twins concordant for response to

clozapine. Lancet, 347, 61.

I. Mata, V. Madoz Fundacion Argibide, Instituto de Salud Mental de Navarra, Apartado de Correos 435, 31080 Pamplona, Spain

M. J. Arranz, P. Sham, R. M. Murray

Department of Psychiatry, Institute of Psychiatry, London

\section{Penile self-mutilation}

Self-injurious behaviour, self-mutilative behaviour or self-harming behaviour are defined as deliberate destruction of body tissue without conscious suicidal intent (Feldman, 1988). An alternative definition of self-injurious behaviour is repetitive, direct physical self-harm that is evidently not life-threatening (Herpertz, 1995). Some other terms such as autoaggression, purposive accidents and focal suicide are also used. The three most commonly reported types of self-injurious behaviour are selfcutting of the skin, ocular self-mutilation and genital self-mutilation (Feldman, 1988). In Greilsheimer \& Groves's (1979) study a majority of cases of male genital self-mutilation had psychosis. Cases of non-psychotic genital self-mutilation include men with character disorders and transsexuality. Many of the patients seemed influenced by religious factors, such as 